\title{
Fine-structural localization of phosphatase activity on the plasma membrane of the rabbit sperm head
}

\author{
Mildred Gordon and Pramila V. Dandekar \\ Anatomical Sciences, State University of New York, Buffalo, New York 14214, U.S.A.
}

The mammalian spermatozoon undergoes a series of maturational changes after leaving the testis. Spermatozoa in the caput epididymidis are not fertilizable (Orgebin-Crist, 1967) but are functional when they reach the cauda. Differentiation of the membranes of caput spermatozoa during epididymal transit was demonstrated by the binding of colloidal iron (Cooper \& Bedford, 1971) and Concanavalin A (Gordon, Dandekar \& Bartoszewicz, 1975). Exposure to seminal plasma appears to stabilize the surface coat (Gordon et al., 1975), the plasma membrane enzymes (Gordon, 1973), and acrosomal enzymes (Zaneveld, Dragoje \& Schumacher, 1972). Spermatozoa become capacitated in the female genital tract, a process which removes seminal substances (Ericsson, 1967; Oliphant \& Brackett, 1973) and modifies the lectin binding of the membrane (Gordon et al., 1975). The segment of the plasma membrane overlying the acrosome of spermatozoa from the cauda epididymidis contains a neutral ATPase which is not active in ejaculates (Gordon, 1973) and it was postulated that the enzyme may become competent during capacitation following removal of seminal antigens.

In the present study, (1) spermatozoa from the caput epididymidis of New Zealand White rabbits were incubated for phosphatase activity to determine whether enzymatic properties of the membrane are developed before epididymal transit; (2) cauda spermatozoa were tested for the effects of seminal plasma by preincubation in seminal plasma from a vasectomized buck before enzyme analysis; and (3) capacitated spermatozoa were incubated for enzymatic activity to monitor for reactivation of the enzyme.

Spermatozoa from the caput epididymidis, preincubated $\left(37^{\circ} \mathrm{C}\right.$ for $\left.30 \mathrm{~min}\right)$ cauda spermatozoa, and uterine-capacitated spermatozoa obtained $15 \mathrm{~h}$ after mating, were fixed in cold buffered $1.5 \%$ (w/v) glutaraldehyde, washed in cold buffer and incubated with ATP and $\mathrm{Mg}^{++}$(Gordon, 1973). Untreated cauda spermatozoa were also incubated with substrate as a control for the procedure. Control media, without substrate, were used simultaneously for each test medium. After incubation, samples were processed for electron microscopy and examined with or without staining with uranyl and lead salts.

The localization of the reaction product in the spermatozoa from the cauda epididymidis was as previously described (Gordon, 1973): reaction product was limited to the periacrosomal membrane and was not present on the post-acrosomal cap or flagellum. There was no reaction visible in any cells from control incubations. Cauda spermatozoa exposed to seminal fluid showed either no activity or great reduction in reaction (Pl. 1, Fig. 2). Such incubations served as an additional control for the specificity of the experimental reaction. As for the cauda spermatozoa, the reaction product seen on all the caput spermatozoa examined was limited to the periacrosomal membrane (Pl. 1, Fig. 1). The enzyme was similarly active in capacitated spermatozoa (Pl. 1, Fig. 3), but not all capacitated cells demonstrated activity.

The results illustrate that the enzyme is established, probably during spermiogenesis, before the development of other membrane features associated with epididymal passage, e.g. increase in Con A receptors (Gordon et al., 1975) and change in negative charge (Nevo, Michaeli \& Schindler, 1961; Cooper \& Bedford, 1971). The absence of enzyme reaction on cauda spermatozoa exposed to seminal plasma lends support to the evidence that seminal constituents, probably decapacitation factors, bind to the sperm head surface. Cauda spermatozoaalso require capacitation, but decapacitating factors in epididymal fluid (Weinman \& Williams, 1964) apparently do not function by inactivation of the membrane enzyme. The lack of activity on many capacitated spermatozoa suggests that spermatozoa in a given ejaculate are not physiologically synchronized. Unreacted spermatozoa may not have been 
sufficiently altered to affect enzyme activity or the activity may have been lost because of cellular degeneration. In a given population of spermatozoa, therefore, some cells may be capacitated at different times, thereby extending the period for interaction with the ovum in the oviduct. Once constituted, the plasmalemmal enzyme may function in collaboration with the $\mathrm{Ca}^{++}$-specific ATPase of the outer acrosome membrane (Gordon, 1973) to promote $\mathrm{Ca}^{++}$activation of acrosomal enzymes (Zaneveld et al., 1972). In other species, such as the guinea-pig and sea urchin, the influx of $\mathrm{Ca}^{++}$ for induction of the acrosome reaction has been shown by use of the ionophore A23187 (Summers, Talbot, Keough, Hylander \& Franklin, 1976). Reactivation of the membrane ATPase during capacitation is probably related to other surface changes (Ericsson, 1967; Oliphant \& Brackett, 1973) such as modification in the receptor population (Gordon et al., 1975).

This research was supported by NICHD grant HD-1003101.

\section{References}

COOPER, G.W. \& Bedford, J.M. (1971) Acquisition of surface change by the plasma membrane of mammalian spermatozoa during epididymal maturation. Anat. Rec. 169, 306, Abstr.

ERICsson, R.J. (1967) A fluorometric method for measurement of sperm capacitation. Proc. Soc. exp. Biol. Med. 125, 1115-1118.

Gordon, M. (1973) Localization of phosphatase activity on the membranes of the mammalian sperm head. J. exp. Zool. 185, 111-120.

Gordon, M., Dandekar, P.V. \& Bartoszewicz, W. (1975) The surface coat of epididymal, ejaculated and capacitated sperm. J. Ultrastruct. Res. 50, 199207.

Nevo, A.C., Michaeli, I. \& SchindleR, H. (1961) Electrophoretic properties of bull and rabbit spermatozoa. Expl Cell Res. 23, 68-83.
Oliphant, G. \& BRackett, B.G. (1973) Immunological assessment of surface changes of rabbit sperm undergoing capacitation. Biol. Reprod. 9, 404 414.

Orgebin-Crist, M.-C. (1967) Sperm maturation in rabbit epididymis. Nature, Lond. 216, 816-818.

Summers, R.G., Talbot, P., Keough, E.M., Hylander, B.L. \& FrankliN, L.E. (1976) Ionophore A23187 induces acrosome reactions in sea urchin and guinea pig spermatozoa. J. exp. Zool. (in press).

Weinman, D.E. \& Williams, W.L. (1964) Mechanism of capacitation of rabbit spermatozoa. Nature, Lond. 203, 423-424.

Zaneveld, L., Dragoje, B.M. \& Schumacher, G.F.B. (1972) Acrosomal proteinase and proteinase inhibitor of human spermatozoa. Science, N.Y. 177, $702-703$. 

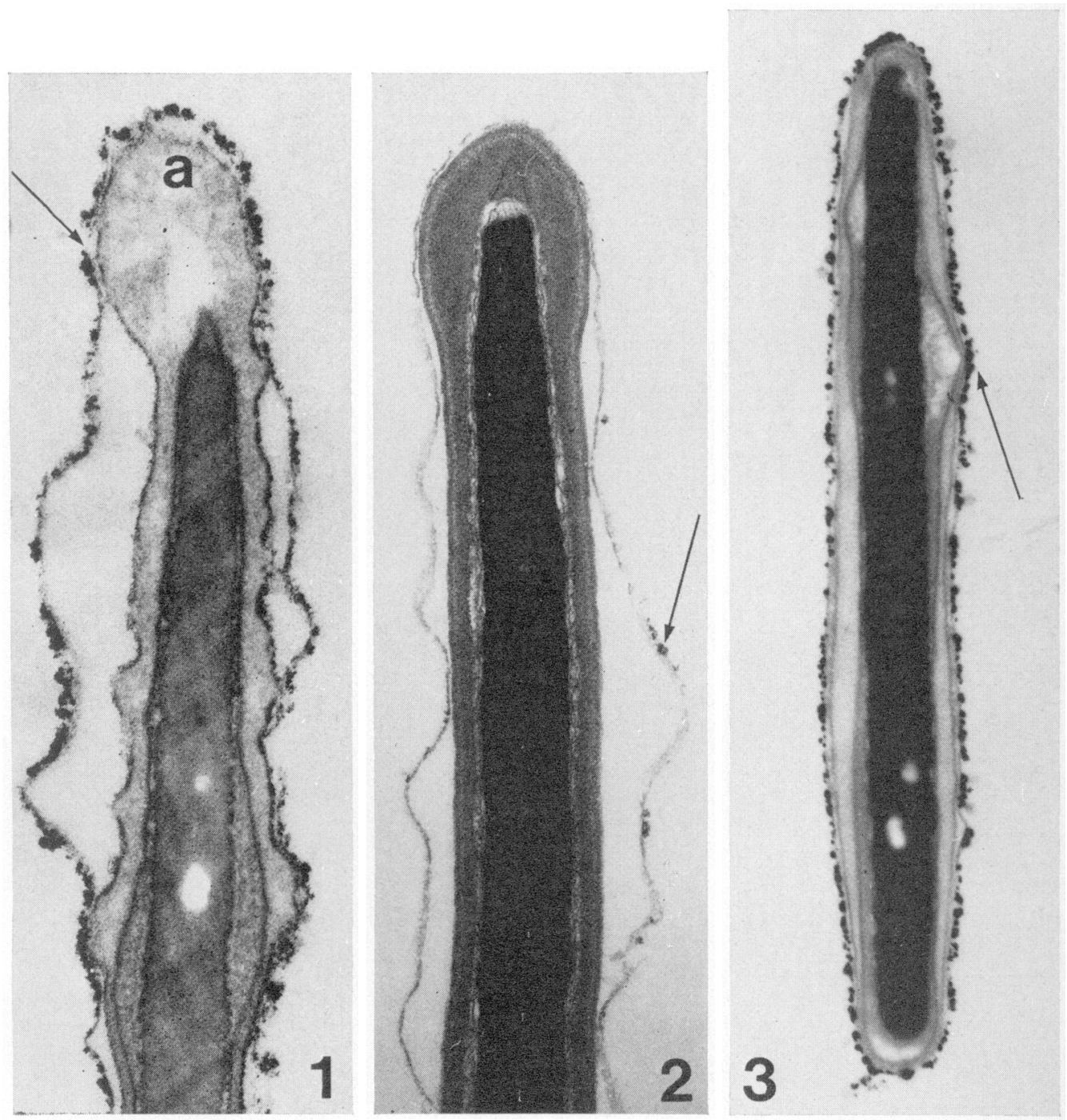

Fig. 1. Spermatozoon from the rabbit caput epididymidis, incubated for ATP, pH $7 \cdot 0$, demonstrating reaction product (arrow) on the plasmalemma over the acrosome (a). $\times 45,900$.

Fig. 2. Cauda spermatozoon, preincubated with seminal plasma before incubation for phosphatase activity. There is no reaction product on the plasma membrane except for occasional, isolated deposits (arrow). $\times 44,800$.

Fig. 3. A capacitated rabbit spermatozoon showing reaction product (arrow) on the plasmalemma after incubation for neutral phosphatase. $\times 30,000$. 